\title{
Recent advances in nanoparticle formulation of oleanolic acid
}

\author{
Meiwan Chen ${ }^{\dagger}$, Zhangfeng Zhong ${ }^{\dagger}$, Wen Tan, Shengpeng Wang and Yitao Wang ${ }^{*}$
}

\begin{abstract}
Oleanolic acid $(\mathrm{OA})$ is a natural triterpenoid possessing anti-inflammatory, antitumor, antiviral, hepatoprotective and antihyperlipidemic effects. Research on the pharmacological activities and clinical applications of OA has made significant progress in the past decade, particularly in the areas such as isolation and purification, chemical modifications, pharmacological research, toxicity studies and clinical use of OA. However, due to its poor aqueous solubility, instability and low bioavailability, OA's clinical applications are still rather limited. Recently, nanoparticulate drug delivery as the biological dimension of nanotechnology has been developed, which may help generate useful formulations of OA for clinical applications. Nanoparticulate drug delivery system enhances the dissolution rate and bioavailability of OA, providing a feasible formulation method for clinical applications.
\end{abstract}

\section{Introduction}

Oleanolic acid (OA), a naturally occurring pentacyclic triterpenoid extracted from the leaves and roots of Olea europaea, Viscum album L., Aralia chinensis L. and over 120 other plant species [1], is chemically known as $3 \beta$ hydroxy-olea-12-en-28-oic acid [2] (Figure 1). OA exhibits many biological activities such as anti-inflammatory, antitumor, antiviral, hepatoprotective and anti-hyperlipidemic effects. OA has been used in Chinese medicine to treat liver disorders for over 20 years [2]. Conventional formulations of OA are tablets and capsules [3]; however, OA's poor aqueous solubility and low bioavailability in vivo make it necessary to develop new formulations for clinical applications.

Derived from nanotechnology, nanoparticulate delivery system provides an innovative approach to drug delivery [4-7]; nanoparticulate technique reduces particles to nanometer ranges, thus reducing the dose and reactive nature of the molecule [8]. Various nanoparticulate drug delivery systems have been explored, such as nanoparticles, nanospheres, nanocapsules, solid lipid nanoparticles (SLN), self-emulsifying drug delivery systems (SEDDS) and submicron/nanoemulsions [9][10]. Compared to conventional dosage forms, nanoparticulate drug delivery system has many advantages, namely enhancement

\footnotetext{
* Correspondence: ytwang@umac.mo

+ Contributed equally

State Key Laboratory of Quality Research in Chinese Medicine, Institute of

Chinese Medical Sciences, University of Macau, Macao SAR, China
}

of solubility and stability, protection from toxicity, enrichment of pharmacological activities, improvement of tissue macrophage distribution, bioavailability and sustained delivery, protection from physical and chemical degradation $[7,11]$.

This article reviews recent advances in nanoparticulate formulation of OA.

\section{Solid lipid nanoparticles}

Solid lipid nanoparticles (SLN), which remain solid at room temperature, have emerged as a new pharmaceutical delivery system or formulation to modify the release profile for many drugs [12]. SLN has characteristics of drug carriers such as lipophilicity, hydrophilicity as well as low bio-toxicity. Main advantages of SLN include: controlling drug release, targeting with reduced toxicity, increasing drug stability and high drug payload [13].

High pressure homogenization is an established method for SLN production. Film-ultrasound dispersion technique is another rational and practicable method for developing a new OA injection [9]. A study showed that the OA solid lipid nanoparticles (OA-SLN) by filmultrasound dispersion technique were with the diameter $(62.0 \pm 10.3)$ (mean \pm standard deviation) $\mathrm{nm}$, encapsulation efficiency (98.29\%), loading rate $(8.17 \%)$ in OASLN [9]. In another study, the researchers prepared OA solid lipid nanoparticles using the optimal preparation conditions (ultrasonic wave time $40 \mathrm{~min}$, OA-phospholipids (1:8), $60 \mathrm{~g} / \mathrm{L}$ mannitol $15 \mathrm{~mL}$ ) by film-ultrasonic 


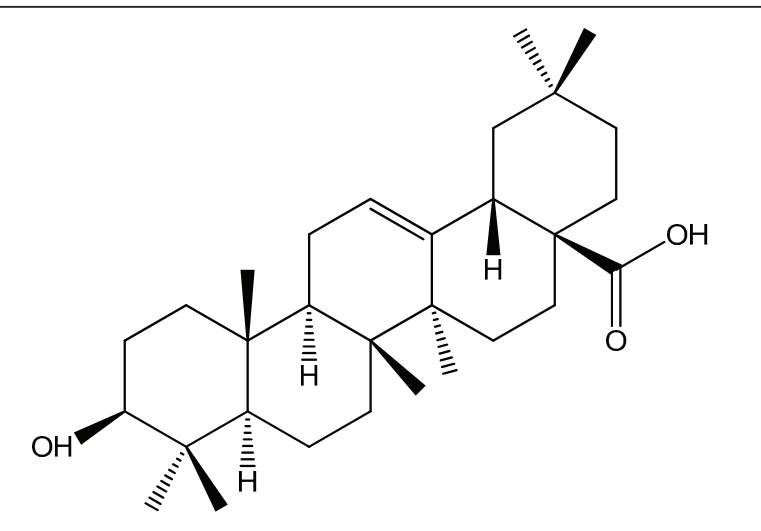

Figure 1 Chemical structrue of oleanolic acid (OA).

wave dispersion technique; the appearance of the prepared solid lipid nanoparticles was regular round or ellipse and the diameter distribution was $(75 \pm 20.3)$ $\mathrm{nm}$; the envelopment ratio was over 97. 81\% [14].

Exploring the protective effect of galactoside - modified OA solid lipid nanoparticles (OA-G10SLN) on $\mathrm{CCl}_{4}$-induced acute hepatic injury of rats in an in vivo study, Wang et al. [15] found that the serum levels of AST and ALT in OA-G10SLN group decreased remarkably compared with a model group, and that the degeneration and necrosis of liver tissues were alleviated significantly, with efficacy better than that in the OA regular solution group.

\section{Nanosuspension}

Nanosuspension technology has been used to increase the solubility, dispersity and homogenization, intravenous injectability, simple production process, universal adaptivity of poorly water soluble drugs [16]. In addition, the formation of suspensions is much more appropriate at low cost and with simple technology to yield a more stable product [16].

There are two major methods for preparing nanosuspension, namely (1) high-pressure homogenization and (2) nanoprecipitation. Homogenization pressure is the major factor determining the average particle size: increased homogenization cycles led to a decreased polydispensible index [17] and surfactants helped keep the system stable [10]. The solubility and dissolution of drug nanoparticles were better than crude drug powder [10]. Researchers obtained OA nanosuspensions with average particle size of $284.9 \mathrm{~nm}$ using this method. Drug in the form of spherical or near-spherical nanoparticles in the nanosuspensions showed a faster drug dissolution rate [18]. Pre-treatment of cells with OA nanosuspensions significantly enhanced the hepatoprotective effect against carbon tetrachloride-induced liver injury through lowering serum alanine aminotransferase (ALT) activity and liver malondialdehyde content [18].

In a formulation study [19], several cryoprotectants were employed to study the protective effects of the freeze-dried OA-loaded nanosuspensions. The optimum formulation was selected according to the mean particle sizes of samples before and after the freeze-drying process. The particles of the best sample achieved a mean particle size of $236.3 \mathrm{~nm}$ and a much higher polydispersity index of 0.242 [19]. The study showed that the optimum lyophilized powder could be obtained with $10 \%$ sucrose as a cryoprotectant.

\section{Nanocapsules}

Loading of drugs into ultrafine host vesicles or colloidal capsules in the nanometer size range was recognized as a technique to optimize controlled drug delivery [20]. Nanocapsules are designed to improve stability, absorption, quantitative tissular transfer and pharmacodynamic activity. Furthermore, they avoided side effects and foreign body irritation with better local and systemic tolerance during and after medication [20].

Dynamic penetration system for sustained OA release from the nanocapsules showed that an HPLC profile curve of the OA loaded nanocapsules fitting the Weibull equation. It was demonstrated that OA loaded nanocapsules sustained the release of OA with a $t_{1 / 2}$ about 6.7 times of the control [21].

\section{Liposomes}

A liposome is a vesicle consisting of a flexible bilayer and surrounded by an aqueous core domain. Liposomes were used to improve the therapeutic activity and safety of drugs for the past few decades. Advantages of liposomes include high biocompatibility, easy preparation, high chemical versatility and simple modulation of their pharmacokinetic properties by changing the chemical composition of the bilayer components [22].

OA liposomes were prepared with film-ultrasound technique; optimal formulation and preparation techniques were selected through a test of orthogonal design and evaluated according to the entrapment rates and confirmed liposomes [23]. Selected formulation and preparation technique of OA liposomes consistently achieved regular liposomes with an average size of 182 $\mathrm{nm}$ and entrapment rate of $92.91 \%$. Chen et al. prepared OA liposomes using ethanol injection-sonication and studied the pharmacokinetics of OA liposomes in rats [17]. OA liposomes were almost spherical with a mean diameter of $(206.4 \pm 4.7) \mathrm{nm}$. The encapsulation efficiency of OA liposomes was over $90 \%$ without hemolyticus. The pharmacokinetic parameters of liposomes were better than those of non-liposomes [17]. 


\section{Proliposomes}

The concept of proliposome was introduced to improve the stability of liposome. Proliposomes are dry, freeflowing particles that immediately form a liposomal suspension when in contact with water [24]. Proliposome technologies can produce liposome on a large scale and replace the thin film method [25].

A new proliposome preparation method was used to trap OA into the liposomes [26]. Particle size of the liposomes was small and uniformly distributed. The entrapment efficiency was $(85.65 \pm 7.96) \%$ and increased when $\mathrm{pH}$ was increased or the proportion of the the proportion of the drug and the phosphatide (P/D) was increased from 5:1 to 10:1. The liposomes increased the small intestinal absorption of the drug as determined by the isolated small intestinal absorption method, showing a larger area under curve (AUC) in serosal fluid of proliposome than that of the control group [27].

\section{Self- microemulsifying drug delivery system}

Composed of oils and surfactants, self-emulsifying drug delivery systems (SEDDS) was reported to have many advantages, especially in enhancing oral bioavailability of poorly absorbed drugs [28]. Ideal isotropic including cosolvents would disperse in the aqueous environment of the gastrointestinal tract to form a fine oil-in-water emulsion under gentle agitation to improve the oral bioavailability of the drug with poor water-solubility [29]. Compared to conventional emulsions, SNEDDS was reported to be a thermodynamically and physically stable formulation with high solubility and offer an improvement in dissolution rates and extents of absorption, resulting in more reproducible blood-time profiles [30].

Recently, OA SNEDDS was formulated with Sefsol 218, Cremophor EL, Labrasol, and Transcutol P by pseudoternary phase diagrams to identify self-emulsification regions for the rational design. A remarkable increase in dissolution was observed for the SNEDDS in comparison with the commercial tablet. Oral absorption of OA from SNEDDS showed a 2.4-fold increase in relative bioavailability. An increased mean retention time of OA in rat plasma was also observed [31]. These results suggest the potentials of SNEDDS in improving dissolution and oral bioavailability for poorly water-soluble triterpenoids. Another study reported the preparation of OA selfmicroemulsion with ethyl oleate/EL-40/alcohol selfmicroemulsion system and quality evaluation of OA selfmicroemulsion with the morphology, particle, diameter distribution, physico-chemical properties and stability [32]. The microemulsion was clear and transparent. The microemulsion vesicles appeared as spherical liquid droplets with a Transmission electron microscopy (TEM) after diluted with average diameter of $49.8 \mathrm{~nm}$. Properties of the microemulsion were stable in the stability test. The authors concluded that the self-microemulsion which improved solubility was easy to prepare. In vitro dissolution and absorption kinetics of OA self-microemulsion were studied with paddle method and in situ perfusion method respectively. Dissolution of OA was significantly increased by self-microemulsifying drug delivery system compared with commercially available tablets [6]. OA self-microemulsifying system significantly enhanced the absorption of $\mathrm{OA}$ in the gastrointestinal tract and improved its bioavailability [33].

\section{Submicron emulsions}

Submicron/nano emulsions are a system of at least two nearly immiscible fluids dispersing one into another in the form of droplets with diameter well below the micron level [34]. Nano/submicron emulsions has drawn much attention from the pharmaceutical, cosmetic and food industries [35]. Submicron/nano emulsions are expected to improve uptake efficiency of lipophilic substances as particle absorption rates in the gastrointestinal tract were correlated to the droplet size [35]. This technology provides colloidal drug carriers for various therapeutic applications such as parenteral, oral, ophthalmic or transdermal delivery systems [36]. Zhao et al. developed and validated a simple yet robust HPLC method for the quantitative determination of OA content and partition coefficient of OA in a submicron emulsionbased formulation [37].

\section{Conclusion}

Nanoparticulate drug delivery system enhances the dissolution rate and bioavailability of OA, providing a feasible formulation method for clinical applications.

\section{Abbreviations}

OA: oleanolic acid; SLN: solid lipid nanoparticles; SEDDS: self-emulsifying drug delivery systems; PI: polydispensible index; P/D: the proportion of the drug and the phosphatide; TEM: transmission electron microscopy; OA-SLN: OA solid lipid nanoparticles

\section{Acknowledgements}

This study was supported by the Macao Science and Technology Development Fund (029/2007/A2) and the Research Fund of the University of Macau (UL016A/09-Y2/CMS/WYT01/ICMS)

\section{Authors' contributions}

MC and ZZ drafted the manuscript. WT and SW coordinated and revised the study. YW reviewed and confirmed this paper. All authors read and approved the final version of the manuscript.

\section{Competing interests}

The authors declare that they have no competing interests.

Received: 29 January 2011 Accepted: 27 May 2011

Published: 27 May 2011

\section{References}

1. Perez-Camino MC, Cert A: Quantitative determination of hydroxy pentacyclic triterpene acids in vegetable oils. J Agric Food Chem 1999, 47:1558-1562. 
2. Wang $X$, Ye XL, Liu R, Chen HL, Bai H, Liang X, Zhang XD, Wang Z, Li WL, Hai CX: Antioxidant activities of oleanolic acid in vitro: Possible role of Nrf2 and MAP kinases. Chem Biol Interact 2010, 184:328-337.

3. $X_{i} J$, Tang $H Y$, Zheng Y: Oral dosage forms of oleanolic acid and their pharmacokinetics. Chin J New Drugs 2009, 18:507-515.

4. Kurmi BD, Kayat J, Gajbhiye V, Tekade RK, Jain NK: Micro- and nanocarriermediated lung targeting. Expert Opin Drug Deliv 2010, 7:781-794.

5. Seigneuric R, Markey L, Nuyten DS, Dubernet C, Evelo CT, Finot E, Garrido C: From nanotechnology to nanomedicine: applications to cancer research. Curr Mol Med 2010, 10:640-652.

6. Yang R, Su LQ, Huang X, Zhai GX: Preparation and in vitro dissolution of oleanolic acid self-microemulsifying drug delivery system. China Pharm 2010, 21:3128-3131.

7. Kumar CSSR: Nanotechnology tools in pharmaceutical R\&D. Mater Today 2010, 12:24-30.

8. Thassu D, Deleers M, Pathak Y: Nanoparticulate drug delivery systems. Int J Pharm 2008, 358:307.

9. Wang JW, Tang HF, Shen M, Wang L, Fang KQ: Preparation and quality evaluation of oleanolic acid-loaded solid lipid nanoparticles. J Fourth MilMed Univ 2007, 28:472-472.

10. Chen YJ, Yang XL, Zhao XL, Xu HB: Preparation of oleanolic acid nanosuspension. Chin Pharm J 2006, 41:924-927.

11. Raffa V, Vittorio O, Riggio C, Cuschieri A: Progress in nanotechnology for healthcare. Minim Invasive Ther Allied Technol 2010, 19:127-135.

12. Souto EB, Doktorovova S: Chapter 6 - Solid lipid nanoparticle formulations pharmacokinetic and biopharmaceutical aspects in drug delivery. Methods Enzymol 2009, 464:105-129.

13. Mei Z, Chen H, Weng T, Yang Y, Yang X: Solid lipid nanoparticle and microemulsion for topical delivery of triptolide. Eur J Pharm Biopharm 2003, 56:189-196.

14. Wang J, Zhang S, Wen A, Tang H, Fang K, Liu D: Optimizing for preparation technique of oleanolic acid solid lipid nanoparticles by orthogonal test. Zong Cao Yao 2007, 38:683-685.

15. Wang J, Wen A, Tang H, Wang L, Liu D: Protective effects of galactosidemodified oleanolic acid solid lipid nanoparticles on CCl4-induced acute hepatic injury in rats. China Pharm 2008, 19:1472-1473.

16. Latha S, Selvamani P, Kumar CS, Sharavanan P, Suganya G, Beniwal VS, Rao PR: Formulation development and evaluation of metronidazole magnetic nanosuspension as a magnetic-targeted and polymericcontrolled drug delivery system. J Magn Magn Mater 2009, 321:1580-1585.

17. Chen H, Niu J, Xiao Y, Chen Z, He P, You G: Pharmacokinetics of oleanolic acid liposomes in rats. Chin Tradit Pat Med 2010, 32:569-572.

18. Chen Y, Liu J, Yang X, Zhao X, Xu H: Oleanolic acid nanosuspensions: preparation, in-vitro characterization and enhanced hepatoprotective effect. J Pharm Pharmacol 2005, 57:259-264.

19. Zhao XL, Chen HB, Chen YJ, Yang XL: Freeze-drying of oleanolic acidloaded nanosuspensions. Zhongquo Zhong Yao Za Zhi 2007, 32:1874-1876.

20. Speiser PP: Nanoparticles and liposomes: a state of the art. Methods Find Exp Clin Pharmacol 1991, 13:337-342.

21. Huang Y, Li Y, Li XZ, Liu S, Lei P, Xiao J: Study on the release of oleanolic acid loaded nanocapsules in vitro. Zhong Yao Cai 2008, 31:283-285.

22. Terreno E, Delli Castelli D, Cabella C, Dastru W, Sanino A, Stancanello J, Tei L, Aime S: Paramagnetic liposomes as innovative contrast agents for magnetic resonance (MR) molecular imaging applications. Chem Biodivers 2008, 5:1901-1912.

23. Lai H, Ma L: Study on preparation of oleanolic acid liposomes. Journal of Xinjiang Medical University 2007, 30:581-583.

24. Xiao Y, Song Y, Chen Z, Ping Q: Preparation of silymarin proliposome: a new way to increase oral bioavailability of silymarin in beagle dogs. Int $J$ Pharm 2006, 319:162-168.

25. Chen CM, Alli D: Use of fluidized bed in proliposome manufacturing. J Pharm Sci 1987, 76:419.

26. Wang M, Gao X: Study on preparation of a new oleanolic acid proliposomes and its properties. Chin Pharm J 2007, 42:839-843.

27. Wang M, Gao X: Study on small intestinal absorption of oleanolic acid new proliposome in rat. Journal of Xinjiang Medical University 2007, 30:122-124.

28. Hong JY, Kim JK, Song YK, Park JS, Kim CK: A new self-emulsifying formulation of itraconazole with improved dissolution and oral absorption. J Control Release 2006, 110:332-338.
29. Zhao Y, Wang C, Chow AH, Ren K, Gong T, Zhang Z, Zheng Y: Selfnanoemulsifying drug delivery system (SNEDDS) for oral delivery of Zedoary essential oil: formulation and bioavailability studies. Int I Pharm 2010, 383:170-177.

30. Wang L, Dong J, Chen J, Eastoe J, Li X: Design and optimization of a new self-nanoemulsifying drug delivery system. J Colloid Interface Sci 2009, 330:443-448.

31. Xi J, Chang $Q$, Chan CK, Meng ZY, Wang GN, Sun JB, Wang YT, Tong HH, Zheng Y: Formulation development and bioavailability evaluation of a self-nanoemulsified drug delivery system of oleanolic acid. AAPS PharmSciTech 2009, 10:172-182.

32. Yang R, Su L, Huang X: Preparation and quality evaluation of oleanolic acid self-microemulsion. Food Drug 2008, 10:40-42.

33. Yang $R$, Su LQ, Huang X: The absorption kinetics of oleanolic acid selfmicroemulsion in rat stomach and intestine. Zhong Yao Cai 2008, 31:1695-1698.

34. Cortes-Munoz M, Chevalier-Lucia D, Dumay E: Characteristics of submicron emulsions prepared by ultra-high pressure homogenisation: Effect of chilled or frozen storage. Food Hydrocolloid 2009, 23:640-654.

35. Nicolaos G, Crauste-Manciet S, Farinotti R, Brossard D: Improvement of cefpodoxime proxetil oral absorption in rats by an oil-in-water submicron emulsion. Int J Pharm 2003, 263:165-171.

36. Grohganz H, Tho I, Brandl M: Development and in vitro evaluation of a liposome based implant formulation for the decapeptide cetrorelix. Eur $J$ Pharm Biopharm 2005, 59:439-448.

37. Zhao YX, Hua HY, Liu L: Development and validation of an HPLC method for determination of oleanolic acid content and partition of oleanolic acid in submicron emulsions. Pharmazie 2009, 64:491-494.

doi:10.1186/1749-8546-6-20

Cite this article as: Chen et al:: Recent advances in nanoparticle formulation of oleanolic acid. Chinese Medicine 2011 6:20.

\section{Submit your next manuscript to BioMed Central and take full advantage of:}

- Convenient online submission

- Thorough peer review

- No space constraints or color figure charges

- Immediate publication on acceptance

- Inclusion in PubMed, CAS, Scopus and Google Scholar

- Research which is freely available for redistribution 